J. Nonlinear Var. Anal. 5 (2021), No. 3, pp. 371-389

Available online at http://jnva.biemdas.com

https://doi.org/10.23952/jnva.5.2021.3.03

\title{
EXISTENCE OF SOLUTIONS OF BILEVEL STRONG VECTOR EQUILIBRIUM PROBLEMS AND THEIR APPLICATIONS
}

\author{
ADELA CAPĂTĂ \\ Department of Mathematics, Technical University of Cluj-Napoca, Cluj-Napoca, România
}

\begin{abstract}
In this paper, a bilevel strong vector equilibrium problem is introduced and investigated by using the Fan's lemma. Two approaches for finding the solutions of this problem are considered. The applications provide sufficient conditions for the existence of the solutions of a Pareto vector optimization over set constraints and the existence of strong vector saddle points for a generalized Nash two-person noncooperative games where the payoff function is vector-valued.
\end{abstract}

Keywords. Strong vector equilibrium problem; Antimonotone operator; $C$-upper semicontinuity; Variational inequality; Strong cone saddle point.

\section{INTRODUCTION}

One of the most remarkable problems studied in the field of nonsmooth analysis is the socalled minimax inequality, established in 1972 by Fan [1], and it consists of:

$$
\text { finding } \bar{x} \in K \text { such that } \varphi(\bar{x}, y) \geq 0 \text {, for all } y \in K \text {, }
$$

where $K$ is a nonempty set of a real topological vector space, and $\varphi: K \times K \rightarrow \mathbb{R}$. Later on, in 1992, Muu and Oettli [2] attributed to the above problem the tag of equilibrium problem (shortly $(E P)$ ), and within this paper particular cases, as optimization problems, variational inequalities and fixed point problems, were described. Further particular cases of $(E P)$, such as, saddlepoint problems, Nash equilibria problems or complementarity problems are taking into account in [3]. Its wide diversity of applications attracted many authors whose published research papers are concerning with the existence and solution methods for finding the equilibria points (see, e.g., $[4,5,6,7,8,9,10]$ and the references therein).

The extension of the scalar equilibrium problem $(E P)$ to vector equilibrium problems can be achieved in different ways. Given a real topological vector space $Y, C \subseteq Y$ a cone, two nonempty sets $K_{1}$ and $K_{2}$, and a bifunction $\varphi: K_{1} \times K_{2} \rightarrow Y$, the following strong vector equilibrium problems can be formulated as:

$$
\begin{aligned}
& \text { find } \bar{x} \in K_{1} \text { such that } \varphi(\bar{x}, y) \notin-C \backslash\left\{0_{Y}\right\} \text {, for all } y \in K_{2} \text {; } \\
& \text { find } \bar{x} \in K_{1} \text { such that } \varphi(\bar{x}, y) \in C \text {, for all } y \in K_{2} \text {. }
\end{aligned}
$$

E-mail address: adela.capata@math.utcluj.ro.

Received March 9, 2021; Accepted April 15, 2021.

(C)2021 Journal of Nonlinear and Variational Analysis 
Whenever the topological interior of the cone $C$ is a nonempty set, we may define the weak vector equilibrium problem:

$$
\text { find } \bar{x} \in K_{1} \text { such that } \varphi(\bar{x}, y) \notin-\operatorname{int} C \text {, for all } y \in K_{2} \text {. }
$$

These vectorial problems were introduced in $[11,12,13]$ and they cover as particular cases vector optimization problems, vector cone saddle point problems, vector variational inequality problems, problems which arise in economics, physics, mechanics and so on. In the last decades, a large number of papers have been devoted to the study of existence results regarding various kinds of solutions for these vector equilibrium problems and their particular cases (see, e.g., $[11,12,14,15,16,17,18,19,20,21])$.

Recently, the equilibrium problems have been generalized and developed in the direction of bilevel equilibrium problems. These bilevel equilibrium problems are actually hierarchical problems where both the upper and the lower level problems are formulated as equilibrium problems. The constraint sets of the upper level problem is taken to be the solution set of other problems, such as, fixed point problems, variational inequalities, optimization problems or equilibrium problems. Moudafi [22] formulated, in 2010, a bilevel equilibrium problem which has been the topic of investigation for many articles since then (see, e.g., [23, 24, 25]). In [26], the authors introduced a bilevel vector equilibrium problem, where both the upper and the lower level problems are given as weak vector equilibrium problems.

The main goal of this paper is to introduce and study a bilevel vector equilibrium problem, where both the upper and the lower level problems are strong vector equilibrium problems. The paper is organized as follows. In Section 2, basic definitions and concepts are recalled. Section 3 is devoted to the study of existence solutions of the considered bilevel vector equilibrium problem. Two approaches are considered. In the first theorem, a scalarization approach is considered, while in the second one, a new cone is defined and this permits us to deal with milder semicontinuity assumptions. As applications, the existence of solutions of a Pareto vector optimization problem over set constraints is investigated in Section 4. The last section, Section 5, obtains existence results for a generalized Nash equilibrium problem.

\section{PRELIMINARIES}

Let $X$ and $Y$ be real Hausdorff topological vector spaces, and $C \subseteq Y$ be a closed convex cone, which defines on $Y$ the partial order relation $\leq_{C}$, i.e., $y_{1} \leq_{C} y_{2}$ iff $y_{2}-y_{1} \in C$. For a nonempty set $M \subseteq X$, by $\operatorname{cl}(\mathrm{M})$, int $(\mathrm{M}), \operatorname{co}(\mathrm{M})$, we denote the closure, the topological interior and the convex hull of $M$. By $Y^{*}$, we denote the dual space of $Y$, and by $C^{*}$, the dual cone of $C$, which is defined as

$$
C^{*}=\left\{y^{*} \in Y^{*} \mid y^{*}(c) \geq 0 \text { for all } c \in C\right\},
$$

while the quasi-interior of the dual cone is

$$
C^{\sharp}=\left\{y^{*} \in Y^{*} \mid y^{*}(c)>0 \text { for all } c \in C \backslash\left\{0_{Y}\right\}\right\} .
$$

Whenever $C$ is a nontrivial convex cone in a locally convex space, $C^{\sharp} \neq \emptyset$ if and only if $C$ admits a base $\mathscr{B}$ and $0_{Y} \notin \operatorname{cl}(\mathscr{B})$ ([27]).

The following concepts represent a generalization of the upper semicontinuity of scalar functions to vector-valued functions.

Definition 2.1. ([28]) A function $F: M \rightarrow Y$ is said to be: 
(i) $C$-upper semicontinuous at a point $\bar{x} \in M$ if, for any neighbourhood $V$ of $F(\bar{x})$, there exists a neighbourhood $U$ of $\bar{x}$ such that

$$
F(x) \in V-C, \text { for all } x \in U \cap M .
$$

(ii) $C$-upper semicontinuous on $M$ if it is $C$-upper semicontinuous at each point $\bar{x} \in M$.

(iii) $C$-lower semicontinuous at $\bar{x} \in M$ if $-F$ is $C$-upper semicontinuous at $\bar{x}$.

(iv) $C$-lower semicontinuous on $M$ if $-F$ is $C$-upper semicontinuous on $M$.

Under the hypothesis that $C$ is a solid convex cone, i.e., int $C \neq \emptyset$, it was shown in [21] that condition (i) is equivalent to:

(v) for each $c \in \operatorname{int} C$, there exists a neighbourhood $U$ of $\bar{x}$ such that

$$
F(x) \in F(\bar{x})+c-\operatorname{int} C, \text { for all } x \in U \cap M .
$$

This condition (v) was already used in [29] to characterize upper semicontinuous vector-valued functions. More precisely, they proved that (v) is equivalent to the following condition:

(vi) for each $c \in \operatorname{int} C$, there exists a neighbourhood $U$ of $\bar{x}$ such that

$$
F(x)-F(\bar{x}) \leq_{C} c \text {, for all } x \in U \cap M .
$$

A quite recent generalization of the ordinary upper semicontinuity of real-valued functions can be found in $[30,31]$.

Definition 2.2. A function $F: M \rightarrow Y$ is said to be:

(i) properly $C$-upper semicontinuous at a point $\bar{x} \in M$ if, for each $c \in C \backslash\left\{0_{Y}\right\}$, there exists $U$ a neighbourhood of $\bar{x}$ such that

$$
F(x) \in F(\bar{x})+c-C \backslash\left\{0_{Y}\right\}, \text { for all } x \in U \cap M .
$$

(ii) properly $C$-upper semicontinuous on $M$ if it is properly $C$-upper semicontinuous at each point $\bar{x} \in M$.

(iii) properly $C$-lower semicontinuous at $\bar{x} \in M$ if $-F$ is properly $C$-upper semicontinuous at $\bar{x}$.

(iv) properly $C$-lower semicontinuous on $M$ if $-F$ is properly $C$-upper semicontinuous on $M$.

For this concept, the forthcoming characterization in terms of level sets holds.

Proposition 2.1. Given a function $F: M \rightarrow Y$, the following properties are equivalent:

(i) $F$ is properly $C$-upper semicontinuous on $M$.

(ii) For each $y \in Y$, the set $F^{-1}\left(y-C \backslash\left\{0_{Y}\right\}\right)$ is open with respect to the induced topology on $M$.

Definition 2.3. A bifunction $G: M \times M \rightarrow Y$ is said to be:

(i) $C$-convex in its second argument if, $M$ is convex, and, for all $x \in M$ the function $G(x, \cdot)$ is $C$-convex, i.e.,

$$
G\left(x, t y_{1}+(1-t) y_{2}\right) \leq_{C} t G\left(x, y_{1}\right)+(1-t) G\left(x, y_{2}\right), \text { for all } y_{1}, y_{2} \in M \text { and } t \in[0,1] .
$$

(ii) $C$-concave in its first argument if $M$ is convex, and, for all $y \in M$, the function $-G(\cdot, y)$ is $C$-convex, i.e.,

$$
t G\left(x_{1}, y\right)+(1-t) G\left(x_{2}, y\right) \leq_{C} G\left(t x_{1}+(1-t) x_{2}, y\right), \text { for all } x_{1}, x_{2} \in M \text { and } t \in[0,1] .
$$


(iii) $C$-monotone if, for all $x, y \in M$,

$$
G(x, y)+G(y, x) \in-C .
$$

(iv) $C$-antimonotone if $-G$ is $C$-monotone, i.e., for all $x, y \in M$,

$$
G(x, y)+G(y, x) \in C .
$$

Definition 2.3 (i) can be found in [32], while (iv) represents a generalization of the concept of the antimonotonicity coming from scalar bifunctions. The term "anti" appears in the context of "cyclic antimonotonicity" of [33]. The following cornerstone result of nonlinear analysis was established by Fan in [34], and it deals with the well-known concept of the Knaster-KuratowskiMazurkiewicz operator.

Definition 2.4. The set-valued mapping $T: M \rightarrow 2^{M}$ is said to be a KKM-operator if, for every finite subset $\left\{x_{1}, x_{2}, \ldots, x_{n}\right\}$ of $M$,

$$
\operatorname{co}\left\{x_{1}, x_{2}, \ldots, x_{n}\right\} \subseteq \bigcup_{i=1}^{n} T\left(x_{i}\right) .
$$

Lemma 2.1 ([34]). Let $T: M \rightarrow 2^{M}$ be a KKM-operator satisfying the following conditions:

(i) $T(x)$ is a closed set for all $x \in M$;

(ii) there is $\bar{x} \in M$ such that $T(\bar{x})$ is a compact set.

Then,

$$
\bigcap_{x \in M} T(x) \neq \emptyset
$$

Next, the property of essentially quasimonotonicity of a scalar bifunction, and a generalization of it to vector bifunctions are recalled.

Definition 2.5 ([35]). Let $M$ be a convex set. A bifunction $G: M \times M \rightarrow \mathbb{R}$ is said to be essentially quasimonotone iff, for arbitrarily integer $n \geq 1$, for every $x_{1}, \ldots, x_{n} \in M$ and $\lambda_{1}, \ldots, \lambda_{n} \geq 0$ such that $\sum_{i=1}^{n} \lambda_{i}=1$, it holds that

$$
\sum_{i=1}^{n} G\left(x_{i}, \sum_{j=1}^{n} \lambda_{j} x_{j}\right) \leq 0 .
$$

Definition 2.6 ([36]). Let $M$ be a convex set, and int $C \neq \emptyset$. A bifunction $G: M \times M \rightarrow \mathbb{Y}$ is said to be $C$-essentially quasimonotone iff, for arbitrarily integer $n \geq 1$, for every $x_{1}, \ldots, x_{n} \in M$ and $\lambda_{1}, \ldots, \lambda_{n} \geq 0$ such that $\sum_{i=1}^{n} \lambda_{i}=1$, it holds that

$$
\sum_{i=1}^{n} G\left(x_{i}, \sum_{j=1}^{n} \lambda_{j} x_{j}\right) \notin \operatorname{int} C .
$$

Lemma 2.2. ([36, Lemma 3]) Let $M$ be a convex set, int $C \neq \emptyset$, and suppose that the bifunctions $\varphi, \psi: M \times M \rightarrow Y$ satisfy:

(i) $\psi$ is $C$-essentially quasimonotone and $C$-lower semicontinuous in the second argument;

(ii) $\varphi$ is $C$-upper semicontinuous in the first argument, $C$-convex in the second argument, and $\varphi(x, x) \in C$ for all $x \in M$. 
Then there exists $\bar{x} \in M$ such that

$$
\varphi(\bar{x}, y)-\psi(y, \bar{x}) \notin-\operatorname{int} C,
$$

for all $y \in M$.

Lemma 2.3. ([36, Lemma 4]) Let $M$ be a convex set, int $C \neq \emptyset$, and suppose that the bifunctions $\varphi, \psi: M \times M \rightarrow Y$ satisfy:

(i) $\psi$ is $C$-convex in the second argument, $\psi(x, x) \in C$ for all $x \in M$, and for all $x, y \in M$ the function $t \in[0,1] \mapsto \psi(t y+(1-t) x, y)$ is $C$-upper semicontinuous at 0 ;

(ii) $\varphi$ is $C$-convex in the second argument, and $\varphi(x, x)=0$ for all $x \in M$.

If there exists $\bar{x} \in M$ such that $\varphi(\bar{x}, y)-\psi(y, \bar{x}) \notin-$ int $C$, for all $y \in M$, then

$$
\varphi(\bar{x}, y)+\psi(\bar{x}, y) \notin-\text { int } C, \text { for all } y \in M .
$$

\section{MAIN RESUlTS}

Let $K$ be a nonempty subset of $X$ and let $\Phi, \psi: K \times K \rightarrow Y$ be given vector-valued functions. We consider the following bilevel strong vector equilibrium problem:

$$
(B S V E P) \text { find } \bar{x} \in S_{\psi} \text { such that } \Phi(\bar{x}, y) \notin-C \backslash\left\{0_{Y}\right\} \text {, for all } y \in S_{\psi},
$$

where $S_{\psi}$ denotes the set of solutions of the following strong vector equilibrium problem

$$
(S V E P) \text { find } \bar{z} \in K \text { such that } \psi(\bar{z}, z) \notin-C \backslash\left\{0_{Y}\right\} \text { for all } z \in K \text {. }
$$

In what follows, using a Thikhonov regularization procedure, two existence results for solutions of $(S B V E P)$ will be presented. Firstly, a scalarization approach is considered and the existence of a functional $c^{*} \in C^{\sharp}$, which plays an outstanding impact in the essential quasimonotonicity hypothesis, is required.

Theorem 3.1. Let $K$ be a convex and compact set, $c^{*} \in C^{\sharp}$, and suppose that the following conditions are satisfied:

(i) $\Phi$ is properly $C$-upper semicontinuous in its first argument, $C$-convex in its second argument and $\Phi(x, x) \in C$, for all $x \in K$.

(ii) $\psi$ is properly $C$-lower semicontinuous in its second argument, $C$-antimonotone and $\psi(x, x)=0_{Y}$, for all $x \in K$.

(iii) for all $x, y \in K, \psi(x, y) \notin-C \backslash\left\{0_{Y}\right\}$ implies that $\psi(y, x) \in-C$.

(iv) there exists $c^{*} \in C^{\sharp}$ such that $c^{*} \circ \psi$ is essentially quasimonotone.

Then, problem (BSVEP) admits a solution.

Proof. With $c^{*} \in C^{\sharp}$, for any $\varepsilon>0$, the scalar equilibrium problem $(E P)_{\varepsilon, c^{*}}$ is defined as:

$$
\text { finding } \bar{x}_{\varepsilon} \in K \text { such that } c^{*}\left(\psi\left(\bar{x}_{\varepsilon}, y\right)-\varepsilon \Phi\left(y, \bar{x}_{\varepsilon}\right)\right) \geq 0 \text { for all } y \in K \text {. }
$$

Step 1. We prove that, for any $\varepsilon>0$, the scalar equilibrium problem $(E P)_{\varepsilon, c^{*}}$ admits a solution.

Let $\varepsilon>0$. Consider the set-valued mapping $T: K \rightarrow 2^{K}$ to be defined as:

$$
T(y)=\left\{x \in K \mid c^{*}(\varepsilon \Phi(x, y)-\psi(y, x)) \geq 0\right\}, \text { for all } y \in K
$$


Firstly we will show that $T$ is a KKM-operator. For this, let the points $y_{1}, y_{2}, \ldots, y_{n} \in K$ and take $z \in \operatorname{co}\left\{y_{1}, y_{2}, \ldots, y_{n}\right\}$. Thus, there exists $\lambda_{1}, \lambda_{2}, \ldots, \lambda_{n} \geq 0$ with $\sum_{i=1}^{n} \lambda_{i}=1$ such that

$$
z=\sum_{i=1}^{n} \lambda_{i} y_{i}
$$

Suppose that $z \notin T\left(y_{i}\right)$ for all $i \in\{1,2, \ldots, n\}$. Therefore,

$$
c^{*}\left(\varepsilon \Phi\left(x, y_{i}\right)-\psi\left(y_{i}, x\right)\right)<0, \text { for all } i \in\{1,2, \ldots, n\} .
$$

Multiplying the above inequality by $\lambda_{i}$, and summing over $i$ give

$$
\sum_{i=1}^{n} \lambda_{i} c^{*}\left(\varepsilon \Phi\left(x, y_{i}\right)-\psi\left(y_{i}, x\right)\right)<0 .
$$

By assumption (i), the $C$-convexity of $\Phi$ in its second argument, and $\Phi(x, x) \in C$, for all $x \in K$, we have

$$
\varepsilon \Phi(z, z) \leq_{C} \sum_{i=1}^{n} \lambda_{i} \varepsilon \Phi\left(z, y_{i}\right)
$$

where

$$
\sum_{i=1}^{n} \lambda_{i} \varepsilon \Phi\left(z, y_{i}\right) \in \varepsilon \Phi(z, z)+C \subseteq C+C=C .
$$

On the other side, assumption (iv) furnishes that

$$
\sum_{i=1}^{n} \lambda_{i} c^{*}\left(\psi\left(y_{i}, z\right)\right) \leq 0 .
$$

Therefore, relations (3.2) and (3.3) give the inequality:

$$
c^{*}\left(\left(\sum_{i=1}^{n} \lambda_{i} \varepsilon \Phi\left(z, y_{i}\right)-\sum_{i=1}^{n} \lambda_{i} \psi\left(y_{i}, z\right)\right) \geq 0,\right.
$$

which represents a contradiction to (3.1). Thus $T$ is a KKM-operator. Moreover, $y \in T(y)$, for any $y \in K$, because

$$
\varepsilon \Phi(y, y)-\psi(y, y) \in C+\left\{0_{Y}\right\}=C
$$

provides

$$
c^{*}(\varepsilon \Phi(y, y)-\psi(y, y)) \geq 0 .
$$

Fix $y \in K$, and let $\tau>0$. There exists $c_{0} \in C \backslash\left\{0_{Y}\right\}$ such that $\tau=c^{*}\left(c_{0}\right)$. By the properly $C$-upper semicontinuity of the vector-valued function $x \rightarrow \varepsilon \Phi(x, y)-\psi(y, x)$ at $x_{0} \in K$, for this $c_{0} \in C \backslash\left\{0_{Y}\right\}$, we find that there exists $U$ a neighbourhood of $x_{0}$ such that

$$
\varepsilon \Phi(x, y)-\psi(y, x) \in \varepsilon \Phi\left(x_{0}, y\right)-\psi\left(y, x_{0}\right)+c_{0}-C \backslash\left\{0_{Y}\right\}, \quad \forall x \in U .
$$

By applying $c^{*}$ to the above relation, we have

$$
c^{*}(\varepsilon \Phi(x, y)-\psi(y, x))<c^{*}\left(\varepsilon \Phi\left(x_{0}, y\right)-\psi\left(y, x_{0}\right)\right)+\tau, \quad \forall x \in U .
$$

that is, the scalar function $x \rightarrow c^{*}(\varepsilon \Phi(x, y)-\psi(y, x))$ is upper semicontinuous at $x_{0}$. Since $x_{0}$ was an arbitrarily point in $K$, we have that the function is upper semicontinuous on $K$. Therefore, the level set of 0 , i.e.,

$$
\left\{x \in K \mid c^{*}(\varepsilon \Phi(x, y)-\psi(y, x)) \geq 0\right\}
$$


is closed, and thus $T(y)$ is closed for any $y \in K$. Since $K$ is compact, we have that $T(y)$ is a compact subset of $K$. Since all the assumption of Lemma 2.1 are satisfied, it provides that

$$
\bigcap_{y \in K} T(y) \neq \emptyset \text {. }
$$

So, for any $\varepsilon>0$, there exists $\bar{x}_{\varepsilon} \in K$ such that

$$
c^{*}\left(\varepsilon \Phi\left(\bar{x}_{\varepsilon}, y\right)-\psi\left(y, \bar{x}_{\varepsilon}\right)\right) \geq 0, \text { for all } y \in K,
$$

that is, the scalar equilibrium problem $(E P)_{\varepsilon, c^{*}}$ admits a solution.

Step 2. We prove that $\bar{x} \in K$, the cluster point of the sequence $\left(\bar{x}_{\varepsilon}\right)_{\varepsilon>0}$, belongs to $S_{\psi}$, that is,

$$
\psi(\bar{x}, y) \notin-C \backslash\left\{0_{Y}\right\}, \text { for all } y \in K .
$$

By contradiction, suppose that there exists $y_{0} \in K$ such that $\psi\left(\bar{x}, y_{0}\right) \in-C \backslash\left\{0_{Y}\right\}$. This, together with the $C$-antimonotonicity of $\psi$, namely

$$
\psi\left(\bar{x}, y_{0}\right)+\psi\left(y_{0}, \bar{x}\right) \in C,
$$

permits obtaining $\psi\left(y_{0}, \bar{x}\right) \in C \backslash\left\{0_{Y}\right\}$. So, there exists $c_{0} \in C \backslash\left\{0_{Y}\right\}$ such that

$$
c^{*}\left(c_{0}-\psi\left(y_{0}, \bar{x}\right)\right)<0 .
$$

The properly $C$-upper semicontinuity the vector-valued function $x \rightarrow \varepsilon \Phi\left(x, y_{0}\right)-\psi\left(y_{0}, x\right)$ at $\bar{x} \in$ $K$, as we saw before, assures the upper semicontinuity of the scalar function $x \rightarrow c^{*}\left(\varepsilon \Phi\left(x, y_{0}\right)-\right.$ $\left.\psi\left(y_{0}, x\right)\right)$ at $\bar{x}$. Hence, there exists $\delta_{1}>0$ such that, for all $\varepsilon \in\left(0, \delta_{1}\right)$,

$$
c^{*}\left(\varepsilon \Phi\left(\bar{x}_{\varepsilon}, y_{0}\right)-\psi\left(y_{0}, \bar{x}_{\varepsilon}\right)\right)<c^{*}\left(\varepsilon \Phi\left(\bar{x}, y_{0}\right)-\psi\left(y_{0}, \bar{x}\right)\right)+c^{*}\left(\frac{c_{0}}{2}\right) .
$$

As $c^{*}\left(\frac{c_{0}}{2}\right)>0$, there exists $\delta_{2}>0$ such that

$$
c^{*}\left(\varepsilon \Phi\left(\bar{x}, y_{0}\right)\right)<c^{*}\left(\frac{c_{0}}{2}\right), \text { for all } \varepsilon \in\left(0, \delta_{2}\right) .
$$

Taking $\delta=\min \left\{\delta_{1}, \delta_{2}\right\}$, and using (3.5), (3.6) and (3.7), for all $\varepsilon \in(0, \delta)$, we have

$$
\begin{aligned}
c^{*}\left(\varepsilon \Phi\left(\bar{x}_{\varepsilon}, y_{0}\right)-\psi\left(y_{0}, \bar{x}_{\varepsilon}\right)\right) & <c^{*}\left(\varepsilon \Phi\left(\bar{x}, y_{0}\right)-\psi\left(y_{0}, \bar{x}\right)\right)+c^{*}\left(\frac{c_{0}}{2}\right) \\
& <2 c^{*}\left(\frac{c_{0}}{2}\right)-c^{*}\left(c_{0}\right)=0,
\end{aligned}
$$

which contradicts (3.4).

Step 3. In what follows, we show that the cluster point $\bar{x}$ is solution of $(B S V E P)$.

For this, let $y \in S_{\psi}$. Thus,

$$
\psi(y, z) \notin-C \backslash\left\{0_{Y}\right\} \text { for all } z \in K .
$$

In particular, this holds for all the terms of the sequence $\left(\bar{x}_{\varepsilon}\right)_{\varepsilon>0} \in K$, namely, for any $\varepsilon>0$,

$$
\psi\left(y, \bar{x}_{\varepsilon}\right) \notin-C \backslash\left\{0_{Y}\right\} .
$$

Take $\varepsilon>0$. Using assumption (iii) and the above relation, we have

$$
\psi\left(\bar{x}_{\varepsilon}, y\right) \in-C .
$$

Assert that $\varepsilon \Phi\left(\bar{x}_{\mathcal{E}}, y\right) \notin-C \backslash\left\{0_{Y}\right\}$. By contradiction, suppose that $\varepsilon \Phi\left(\bar{x}_{\varepsilon}, y\right) \in-C \backslash\left\{0_{Y}\right\}$. By this and relation (3.8), we have

$$
\varepsilon \Phi\left(\bar{x}_{\varepsilon}, y\right)+\psi\left(\bar{x}_{\varepsilon}, y\right) \in-C \backslash\{0\}
$$


with

$$
c^{*}\left(\varepsilon \Phi\left(\bar{x}_{\varepsilon}, y\right)+\psi\left(\bar{x}_{\varepsilon}, y\right)\right)<0 .
$$

Because $\psi$ is $C$-antimonotone, we have that there exists an element $c_{\varepsilon} \in C$ such that

$$
\psi\left(y, \bar{x}_{\varepsilon}\right)+\psi\left(\bar{x}_{\varepsilon}, y\right)=c_{\varepsilon} .
$$

Hence, $\psi\left(y, \bar{x}_{\varepsilon}\right)=c_{\varepsilon}-\psi\left(\bar{x}_{\varepsilon}, y\right)$. Replacing it into relation (3.4), we have

$$
c^{*}\left(\varepsilon \Phi\left(\bar{x}_{\varepsilon}, y\right)-c_{\varepsilon}+\psi\left(\bar{x}_{\varepsilon}, y\right)\right) \geq 0,
$$

and

$$
c^{*}\left(\varepsilon \Phi\left(\bar{x}_{\varepsilon}, y\right)+\psi\left(\bar{x}_{\varepsilon}, y\right)\right) \geq c^{*}\left(c_{\varepsilon}\right) \geq 0,
$$

which reach a contradiction to (3.9). As $\varepsilon \Phi\left(\bar{x}_{\varepsilon}, y\right) \notin-C \backslash\left\{0_{Y}\right\}$, we get

$$
\Phi\left(\bar{x}_{\varepsilon}, y\right) \notin-C \backslash\left\{0_{Y}\right\} .
$$

Since $\Phi$ is properly $C$-upper semicontinuous in its first argument at $\bar{x}$, for any $c \in C \backslash\left\{0_{Y}\right\}$, we have that there exist $U_{c}$ a neighbourhood of $\bar{x}$, and $\delta_{c}>0$ such that

$$
\Phi\left(\bar{x}_{\varepsilon}, y\right) \in \Phi(\bar{x}, y)+c-C \backslash\left\{0_{Y}\right\}, \text { for all } \varepsilon \in\left(0, \delta_{c}\right) .
$$

By this relation and (3.10), we conclude that

$$
\Phi\left(\bar{x}_{\varepsilon}, y\right)-\Phi\left(\bar{x}_{\varepsilon}, y\right)+\Phi(\bar{x}, y)+c \notin-C \backslash\left\{0_{Y}\right\} \text {, for all } \varepsilon \in\left(0, \delta_{c}\right) .
$$

Thus,

and

$$
\Phi(\bar{x}, y)+c \notin-C \backslash\left\{0_{Y}\right\}, \text { for all } c \in C \backslash\left\{0_{Y}\right\}
$$

$$
\left(\Phi(\bar{x}, y)+C \backslash\left\{0_{Y}\right\}\right) \cap\left(-C \backslash\left\{0_{Y}\right\}\right)=\emptyset .
$$

If $\Phi(\bar{x}, y) \in-C \backslash\left\{0_{Y}\right\}$, then there exists $c_{0} \in C \backslash\left\{0_{Y}\right\}$ such that

$$
\Phi(\bar{x}, y)+c_{0} \in-C \backslash\left\{0_{Y}\right\},
$$

which reaches a contradiction to (3.11). Therefore, $\bar{x} \in S_{\psi}$ is solution of (BSVEP).

Under the hypothesis that $C^{\sharp} \neq \emptyset$, the following corollary permits dealing only with the assumptions on the involved vector bifunctions of $(B S V E P)$, and with no scalarized conditions.

Corollary 3.1. Let $K$ be a convex and compact set, $C^{\sharp} \neq \emptyset$, and suppose that the following conditions are satisfied:

(i) $\Phi$ is properly $C$-upper semicontinuous in its first argument, $C$-convex in its second argument and $\Phi(x, x) \in C$, for all $x \in K$.

(ii) $\psi$ is properly $C$-lower semicontinuous and $C$-convex in its second argument, $C$-antimonotone, $C$-concave in its first argument and $\psi(x, x)=0$, for all $x \in K$.

(iii) for all $x, y \in K, \psi(x, y) \notin-C \backslash\left\{0_{Y}\right\}$ implies that $\psi(y, x) \in-C$.

Then, (BSVEP) admits a solution.

Proof. Since $C^{\sharp} \neq \emptyset$, there exists $c^{*} \in C^{\sharp}$. According to Theorem 3.1, it remains to prove that assumption (iv) of it is satisfied. For this, let $n \geq 1, x_{1}, \ldots, x_{n} \in K, \lambda_{1}, \ldots, \lambda_{n} \geq 0$ such that $\sum_{i=1}^{n} \lambda_{i}=1$ and $z=\sum_{j=1}^{n} \lambda_{j} x_{j}$. The $C$-concavity of $\psi$ in its first argument, i.e.,

$$
\sum_{i=1}^{n} \lambda_{i} \psi\left(x_{i}, z\right) \leq_{C} \psi(z, z)
$$


which together with $\psi(x, x)=0$, for all $x \in K$, gives

$$
\sum_{i=1}^{n} \lambda_{i} \psi\left(x_{i}, z\right) \in \psi(z, z)-C=-C
$$

Therefore,

$$
\sum_{i=1}^{n} \lambda_{i} c^{*}\left(\psi\left(x_{i}, z\right)\right) \leq 0
$$

and $c^{*} \circ \psi$ is essentially quasimonotone. The conclusion follows now by Theorem 3.1.

The set of solutions of $(B S V E P)$ may be a much smaller subset that the set of solutions of the upper level problem without a constraint set, as the next example shows.

Example 3.1. Let $K=[0,1], Y=\mathbb{R}^{2}, C=\{(x, x) \mid x \geq 0\}$, and consider the bifunctions $\Phi, \psi$ : $K \times K \rightarrow \mathbb{R}^{2}$ to be defined as:

$$
\Phi(x, y)=(x+y, x+y) \text { and } \psi(x, y)=(x-y, x-y) \text {, for all } x, y \in K .
$$

The bifunction $\Phi$ is $C$-convex in its second argument and $\psi(x, x)=(2 x, 2 x) \in C$, for all $x \in K$. For the properly $C$-upper semicontinuity of $\Phi$ in its first argument, fix $y \in K$, and let $x_{0} \in K$ and $c \in C \backslash\left\{0_{2}\right\}$. Thus, there exists $\varepsilon>0$ such that $c=(\varepsilon, \varepsilon)$. Any $x \in U \cap K$, with $U=\left(x_{0}-\frac{\varepsilon}{2}, x_{0}+\frac{\varepsilon}{2}\right)$ verifies:

$$
\Phi(x, y) \in \Phi\left(x_{0}, y\right)+c-C \backslash\left\{0_{2}\right\},
$$

and since $x_{0}$ was arbitrarily chosen, bifunction $\Phi$ is properly $C$-upper semicontinuous in its first argument. In a similar way, the properly $C$-lower semicontinuity of $\psi$ in its second argument can be checked. Since

$$
\psi(x, y)+\psi(y, x)=(0,0) \in C \cap(-C), \text { for all } x, y \in K,
$$

we see that $\psi$ is $C$-antimonotone and $C$-monotone in the same time. As $\psi$ is $C$-monotone and $C$-convex in its second argument, by the proof of [36, Proposition 1], for $c^{*}=(1,1)$, the scalar bifunction $c^{*} \circ \psi$ is essentially quasimonotone.

It remains to verify assumption (iii) of Theorem 3.1. Let $x, y \in K$ and suppose that

$$
\psi(x, y) \notin-C \backslash\left\{0_{2}\right\} \text {, i.e. }(x-y, x-y) \notin-C \backslash\left\{0_{2}\right\},
$$

whence $x-y \geq 0$. Then,

$$
\psi(y, x)=(y-x, y-x) \in-C .
$$

It is easy to observe that $S_{\psi}=\{1\}$, and $\bar{x}=1$ is the only solution of (BSVEP), while the upper level problem without a constraint set (namely, $\psi \equiv 0$ ) admits $K$ as the solution set.

A second approach can be achieved by taking a nonzero functional $c^{*} \in C^{\sharp}$, which allows us to construct the following Henig dilating cone

$$
H=\left\{y \in Y \mid c^{*}(y)>0\right\} \cup\left\{0_{Y}\right\} .
$$

This cone has the property that int $H=H \backslash\left\{0_{Y}\right\}$ and, it is easy to see that $C \backslash\left\{0_{Y}\right\} \subseteq \operatorname{int} H$. For any $\varepsilon>0$, the next auxiliary weak vector equilibrium problem is considered:

$(W V E P)_{\varepsilon, H}$ find $\bar{x}_{\varepsilon} \in K$ such that $\varepsilon \Phi\left(\bar{x}_{\varepsilon}, y\right)+\psi\left(\bar{x}_{\varepsilon}, y\right) \notin-\operatorname{int} H$ for all $y \in K$.

$S_{\varepsilon, H}$ denotes the set of solutions for this problem. This auxiliary equilibrium problem allows us to deal with milder semicontinuity assumptions. 
Theorem 3.2. Let $K$ be a convex and compact set, $c^{*} \in C^{\sharp}$, and suppose that the following conditions are satisfied:

(i) $\Phi$ is $C$-upper semicontinuous in its first argument, $C$-convex in its second argument and $\Phi(x, x)=0$, for all $x \in K$.

(ii) $\psi$ is $C$-lower semicontinuous in its second argument, $C$-monotone, $\psi(x, x)=0$, and, for all $x, y \in M$ the function $t \in[0,1] \mapsto \psi(t y+(1-t) x, y)$ is $C$-upper semicontinuous at 0 .

(iii) for all $x, y \in K, \psi(x, y) \notin-C \backslash\left\{0_{Y}\right\}$ implies that $\psi(y, x) \in-C$.

(iv) there exists $c^{*} \in C^{\sharp}$ such that $c^{*} \circ \psi$ is essentially quasimonotone.

Then, problem (BSVEP) admits a solution.

Proof. With the aid of the functional $c^{*} \in C^{\sharp}$ from hypothesis (iv), we construct the Henig dilating cone $H=\left\{y \in Y \mid c^{*}(y)>0\right\} \cup\left\{0_{Y}\right\}$ and consider the weak vector equilibrium problem:

$(W V E P)_{\varepsilon, H}$ find $\bar{x}_{\varepsilon} \in K$ such that $\varepsilon \Phi\left(\bar{x}_{\varepsilon}, y\right)+\psi\left(\bar{x}_{\varepsilon}, y\right) \notin-\operatorname{int} H$, for all $y \in K$,

which is given as the sum of two bifunctions.

Step 1 . We prove that, for any $\varepsilon>0,(W V E P)_{\varepsilon, H}$ admits a solution.

By assumption (iv), there exists $c^{*} \in C^{\sharp}$ such that $c^{*} \circ \psi$ is essentially quasimonotone. Since the functional $c^{*} \in H^{*} \backslash\left\{0_{Y}^{*}\right\}$, together with $c^{*} \circ \psi$ is essentially quasimonotone, i.e. for arbitarily integer $n \geq 1$, for every $x_{1}, \ldots, x_{n} \in M$ and $\lambda_{1}, \ldots, \lambda_{n} \geq 0$ such that $\sum_{i=1}^{n} \lambda_{i}=1$

$$
\sum_{i=1}^{n} c^{*}\left(\psi\left(x_{i}, \sum_{j=1}^{n} \lambda_{j} x_{j}\right)\right) \leq 0,
$$

it assures that

$$
\sum_{i=1}^{n} \psi\left(x_{i}, \sum_{j=1}^{n} \lambda_{j} x_{j}\right) \notin H \backslash\left\{0_{Y}\right\}=\operatorname{int} H .
$$

Hence, $\psi$ is $H$-essentially quasimonotone.

As $C \subseteq H$, Definition 2.1 and hypothesis (i) and (ii) give that $\Phi$ is $H$-upper semicontinuous in its first argument, respectively, and $\psi$ is $H$-lower semicontinuous in its second argument. Moreover, $\Phi$ is $H$-convex in the second argument and $\Phi(x, x)=0_{Y} \in H$, for all $x \in K$. Applying Lemma 2.2, there exists $\bar{x}_{\mathcal{E}} \in K$ such that

$$
\varepsilon \Phi\left(\bar{x}_{\varepsilon}, y\right)-\psi\left(y, \bar{x}_{\varepsilon}\right) \notin-\operatorname{int} H \text {, for all } y \in K .
$$

Since $\Phi$ and $\psi$ satisfy all the assumptions of Lemma 2.3, we see from relation (3.12) that there exists $\bar{x}_{\varepsilon} \in K$ such that

$$
\varepsilon \Phi\left(\bar{x}_{\mathcal{E}}, y\right)+\psi\left(\bar{x}_{\mathcal{E}}, y\right) \notin-\operatorname{int} H, \text { for all } y \in K,
$$

that is, $(W V E P)_{\varepsilon, H}$ admits a solution.

Step 2. The cluster point $\bar{x} \in K$ of the sequence $\left(\bar{x}_{\varepsilon}\right)_{\varepsilon>0}$ belongs to $S_{\psi}$. Firstly, we prove that

$$
\psi(y, \bar{x}) \notin \operatorname{int} H, \text { for all } y \in K .
$$

By contradiction, suppose that there exists $y_{0} \in K$ such that $\psi\left(y_{0}, \bar{x}\right) \in \operatorname{int} H$. Therefore, there exists $h \in \operatorname{int} H$ such that

$$
h-\psi\left(y_{0}, \bar{x}\right) \in-\operatorname{int} H .
$$


Since $\frac{h}{2}-\operatorname{int} H$ is an open set and $0_{Y} \in \frac{h}{2}-\operatorname{int} H$, there exists $\delta_{1}>0$ such that

$$
\varepsilon \Phi\left(\bar{x}_{\varepsilon}, y_{0}\right) \in \frac{h}{2}-\operatorname{int} H, \text { for all } \varepsilon \in\left(0, \delta_{1}\right) .
$$

Assumptions (i) and (ii) provide that the function $x \in K \mapsto \varepsilon \Phi\left(x, y_{0}\right)-\psi\left(y_{0}, x\right)$ is $C$-upper semicontinuous, and implicitly $H$-upper semicontinuous. Consequently there exist $U$ a neighbourhood of $\bar{x}$ and $\delta_{2}>0$ such that $\bar{x}_{\varepsilon} \in U$, for all $\varepsilon \in\left(0, \delta_{2}\right)$ and

$$
\varepsilon \Phi\left(\bar{x}_{\varepsilon}, y_{0}\right)-\psi\left(y_{0}, \bar{x}_{\varepsilon}\right) \in \varepsilon \Phi\left(\bar{x}, y_{0}\right)-\psi\left(y_{0}, \bar{x}\right)+\frac{h}{2}-\operatorname{int} H, \text { for all } \varepsilon \in\left(0, \delta_{2}\right) .
$$

Taking $\delta=\min \left\{\delta_{1}, \delta_{2}\right\}$, and using relations (3.15), (3.16) and (3.17), we obtain that

$$
\varepsilon \Phi\left(\bar{x}_{\varepsilon}, y_{0}\right)-\psi\left(y_{0}, \bar{x}_{\varepsilon}\right) \in-\text { int } H, \text { for all } \varepsilon \in(0, \delta) .
$$

The monotonicity of $\psi$, which conducts to $\psi\left(y_{0}, \bar{x}_{\varepsilon}\right)+\psi\left(\bar{x}_{\varepsilon}, y_{0}\right) \in-C \subseteq-H$, and the above relation yield

$$
\varepsilon \Phi\left(\bar{x}_{\varepsilon}, y_{0}\right)+\psi\left(\bar{x}_{\varepsilon}, y_{0}\right) \in-\text { int } H, \text { for all } \varepsilon \in(0, \delta),
$$

which reaches a contradiction to (3.13).

Suppose now, that $\bar{x} \notin S_{\psi}$, i.e., there exists $y_{0} \in K$ such that $\psi\left(\bar{x}, y_{0}\right) \in-C \backslash\left\{0_{Y}\right\}$. Therefore,

$$
\psi\left(\bar{x}, y_{0}\right) \in-\operatorname{int} H
$$

and there exists $h \in \operatorname{int} H$ such that

$$
\psi\left(\bar{x}, y_{0}\right)+h \in-\operatorname{int} H .
$$

By hypothesis (iii), the function $t \in[0,1] \mapsto \psi\left(t y_{0}+(1-t) \bar{x}, y_{0}\right)$ is $C$-upper semicontinuous at 0 , and implicitly $H$-upper semicontinuous at 0 . Denote $x_{t}=t y_{0}+(1-t) \bar{x} \in K$. Thus, there exists $\delta_{3} \in(0,1)$ such that

$$
\psi\left(x_{t}, y_{0}\right) \in \psi\left(\bar{x}, y_{0}\right)+h-\operatorname{int} H, \text { for all } t \in\left(0, \delta_{3}\right) .
$$

The above relation, which together with (3.18) gives

$$
\psi\left(x_{t}, y_{0}\right) \in-\operatorname{int} H, \text { for all } t \in\left(0, \delta_{3}\right) .
$$

Besides, $\psi\left(x_{t}, x_{t}\right)=0$, and the $C$-convexity of $\psi$ in the second argument, for all $t \in[0,1]$, yield

$$
t \psi\left(x_{t}, y_{0}\right)+(1-t) \psi\left(x_{t}, \bar{x}\right) \in C \subseteq H .
$$

From this and (3.19), we have

$$
\psi\left(x_{t}, \bar{x}\right) \in \operatorname{int} H, \text { for all } t \in\left(0, \delta_{3}\right),
$$

which is a contradiction to (3.14).

Step 3. The cluster point $\bar{x}$ is a solution of $(B S V E P)$. For this, let $y \in S_{\psi}$, i.e.,

$$
\psi(y, z) \notin-C \backslash\left\{0_{Y}\right\}, \text { for all } z \in K,
$$

and let $\bar{x}_{\varepsilon} \in S_{\varepsilon, H}$. Thus,

$$
\varepsilon \Phi\left(\bar{x}_{\varepsilon}, y\right)+\psi\left(\bar{x}_{\varepsilon}, y\right) \notin-\operatorname{int} H .
$$

By contradiction, we suppose now that $\varepsilon \Phi\left(\bar{x}_{\varepsilon}, y\right) \in-$ int $H$. Relation (3.20) gives

$$
\psi\left(y, \bar{x}_{\varepsilon}\right) \notin-C \backslash\left\{0_{Y}\right\} .
$$


Hypothesis (iii) assures that

$$
\psi\left(\bar{x}_{\varepsilon}, y\right) \in-C
$$

So,

$$
\varepsilon \Phi\left(\bar{x}_{\varepsilon}, y\right)+\psi\left(\bar{x}_{\varepsilon}, y\right) \in-\text { int } H-C \subseteq-\text { int } H,
$$

which contradicts (3.21). Therefore, $\varepsilon \Phi\left(\bar{x}_{\varepsilon}, y\right) \notin-$ int $H$ with

$$
\Phi\left(\bar{x}_{\mathcal{E}}, y\right) \notin-\text { int } H \text {. }
$$

Because $\Phi$ is $C$-upper semicontinuous in its first argument, for any $h \in \operatorname{int} H$, there exist $U$, a neighbourhood of $\bar{x}$, and $\delta_{4}>0$ such that $\bar{x}_{\varepsilon} \in U$, for all $\varepsilon \in\left(0, \delta_{4}\right)$, and

$$
\Phi\left(\bar{x}_{\varepsilon}, y\right) \in \Phi(\bar{x}, y)+h-\operatorname{int} H, \text { for all } \varepsilon \in\left(0, \delta_{4}\right) .
$$

This relation and (3.22) assure

$$
\Phi(\bar{x}, y)+h \notin-\operatorname{int} H, \text { for all } h \in \operatorname{int} H,
$$

which is equivalent to

$$
(\Phi(\bar{x}, y)+\operatorname{int} H) \cap(-\operatorname{int} H)=\emptyset .
$$

If $\Phi(\bar{x}, y) \in-\operatorname{int} H$, there exists $h_{0} \in \operatorname{int} H$ such that

$$
\Phi(\bar{x}, y)+h_{0} \in-\operatorname{int} H
$$

which is a contradiction to (3.23). Therefore, $\Phi(\bar{x}, y) \notin-$ int $H$ implies

$$
\Phi(\bar{x}, y) \notin-C \backslash\left\{0_{Y}\right\},
$$

namely $\bar{x} \in S_{\psi}$ is solution of the problem $(B S V E P)$.

Corollary 3.2. Let $K$ be a convex and compact set, $C^{\sharp} \neq \emptyset$, and suppose that the following conditions are satisfied:

(i) $\Phi$ is $C$-upper semicontinuous in its first argument, $C$-convex in its second argument and $\Phi(x, x)=0$, for all $x \in K$.

(ii) $\psi$ is $C$-lower semicontinuous in its second argument, $C$-monotone, $C$-concave in its first argument, $\psi(x, x)=0$, and, for all $x, y \in M$ the function $t \in[0,1] \mapsto \psi(t y+(1-t) x, y)$ is $C$-upper semicontinuous at 0 .

(iii) for all $x, y \in K, \psi(x, y) \notin-C \backslash\left\{0_{Y}\right\}$ implies that $\psi(y, x) \in-C$.

Then, problem (BSVEP) admits a solution.

Proof. The proof is similar to the one of Corollary 3.1.

\section{PARETo Vector optimization OVER AN EFFicient SET}

Bilevel equilibrium problems include, as particular cases, vector optimization problems over constraint sets. In this section, we investigate existence results for such an hierarchical problem whose upper level problem is a vector optimization problem, while its lower level problem is a Stampacchia vector variational inequality

$$
(B V O P) \min _{x \in S_{T}} \varphi(x),
$$

over $S_{T}$, which denotes the set of solutions of the following Stampacchia strong vector variational inequality:

$(S V I)$ find $\bar{z} \in K$ such that $\langle T(\bar{z}), z-\bar{z}\rangle \notin-C \backslash\left\{0_{Y}\right\}$, for all $z \in K$. 
$\mathscr{L}(X, Y)$ denotes the set of all continuous linear functions from $X$ to $Y$, and $T: K \rightarrow \mathscr{L}(X, Y)$ is an operator. These hierarchical vector optimization problems are also particular cases of bilevel vector optimization problems (see, for instance, [37, 38], and the references therein).

Firstly let us recall some definitions (see, for instance [39, 40, 41])

Definition 4.1. A mapping $T: K \rightarrow \mathscr{L}(X, Y)$ is said to be:

(i) pseudomonotone if, for all $x, y \in K$,

$$
\langle T(x), y-x\rangle \notin-C \backslash\left\{0_{Y}\right\} \text { implies }\langle T(y), y-x\rangle \in C .
$$

(ii) $v$-hemicontinuous if $K$ is a convex set, and, for all $x, y \in K$, the function

$$
\forall t \in[0,1] \mapsto\langle T(t y+(1-t) x), y-x\rangle \in Y
$$

is upper semicontinuous at 0 .

(iii) $C$-monotone if, for all $x, y \in K,\langle T(y)-T(x), y-x\rangle \in C$.

Definition 4.2. A point $\bar{x} \in S_{T}$ is said to be a Pareto efficient solution of (BVOP) if and only if

$$
\varphi(\bar{x}) \in \operatorname{Min}\left(\varphi\left(S_{T}\right), C\right),
$$

i.e., $\left(\varphi\left(S_{T}\right)-\varphi(\bar{x})\right) \cap(-C)=\left\{0_{Y}\right\}$.

Theorem 4.1. Let $K$ be a convex and compact set, $c^{*} \in C^{\sharp}$, and suppose that the following conditions are satisfied:

(i) $\varphi$ is $C$-lower semicontinuous and $C$-convex.

(ii) $T$ is $C$-monotone and v-hemicontinuous.

(iii) $T$ is pseudomonotone.

(iv) there exists $c^{*} \in C^{\sharp}$ such that for arbitarily integer $n \geq 1$, for every $x_{1}, \ldots, x_{n} \in K$ and $\lambda_{1}, \ldots, \lambda_{n} \geq 0$ with $\sum_{i=1}^{n} \lambda_{i}=1$, it holds:

$$
\sum_{i=1}^{n} c^{*}\left(\left\langle T\left(x_{i}\right), \sum_{j=1}^{n} \lambda_{j} x_{j}-x_{i}\right\rangle\right) \leq 0 .
$$

Then, problem (BVOP) admits a solution.

Proof. Consider the functions $\Phi, \psi: K \times K \rightarrow Y$ to be defined as:

$$
\Phi(x, y)=\varphi(y)-\varphi(x) \text {, respectively, } \psi(x, y)=\langle T(x), y-x\rangle \text { for all } x, y \in K .
$$

In what follows, we show that these bifunctions verify the assumptions of Theorem 3.2. It is easy to see that hypothesis (i) provides the $C$-upper semicontinuity of $\Phi$ in its first argument and the $C$-convexity in its second argument. Moreover $\Phi(x, x)=0$, for all $x \in K$.

Bifunction $\psi$ is continuous in its second argument, and therefore, it is $C$-lower semicontinuous in its second argument. $\psi(x, x)=0$, for all $x \in K$ and hypothesis (ii) provides that $\psi$ is $C$-monotone and the function $t \in[0,1] \mapsto \psi(t y+(1-t) x, y)$ is $C$-upper semicontinuous at 0 . Further, hypothesis (iii) and (iv) lead us to assumptions (iii) and (iv) of Theorem 3.2 fulfilled. Hence, all the assumptions of Theorem 3.2 are satisfied and the conclusion follows now by this.

Note that Theorem 4.1 does not guarantee that a Pareto efficient solution over a constraint set becomes a Pareto efficient solution, as the following example illustrates. 
Example 4.1. Let $K=[0,1] \times[0,1], Y=\mathbb{R}^{2}, C=\mathbb{R}_{+}^{2}$, and consider $\varphi: K \rightarrow \mathbb{R}^{2}$ and $T: K \rightarrow$ $\mathscr{L}\left(\mathbb{R}^{2}, \mathbb{R}^{2}\right)$ to be defined as:

$$
\varphi(x)=-x, T(x)=\left(\begin{array}{ll}
x_{1}+x_{2}+1 & x_{1}+x_{2}+1 \\
x_{1}+x_{2}+2 & x_{1}+x_{2}+2
\end{array}\right), \text { for all } x=\left(x_{1}, x_{2}\right) \in K .
$$

We show that all the assumptions from Theorem 4.1 are satisfied. Let $x=\left(x_{1}, x_{2}\right), y=$ $\left(y_{1}, y_{2}\right) \in K$.

$$
\begin{aligned}
\langle T(x), y-x\rangle & =\left(\begin{array}{ll}
x_{1}+x_{2}+1 & x_{1}+x_{2}+1 \\
x_{1}+x_{2}+2 & x_{1}+x_{2}+2
\end{array}\right)\left(\begin{array}{l}
y_{1}-x_{1} \\
y_{2}-x_{2}
\end{array}\right) \\
& =\left(\begin{array}{l}
\left(x_{1}+x_{2}+1\right)\left(y_{1}+y_{2}-x_{1}-x_{2}\right) \\
\left(x_{1}+x_{2}+2\right)\left(y_{1}+y_{2}-x_{1}-x_{2}\right)
\end{array}\right) .
\end{aligned}
$$

Evaluating

$$
\begin{aligned}
\langle T(y)-T(x), y-x\rangle & =\left(\begin{array}{ll}
y_{1}+y_{2}-x_{1}-x_{2} & y_{1}+y_{2}-x_{1}-x_{2} \\
y_{1}+y_{2}-x_{1}-x_{2} & y_{1}+y_{2}-x_{1}-x_{2}
\end{array}\right)\left(\begin{array}{l}
y_{1}-x_{1} \\
y_{2}-x_{2}
\end{array}\right) \\
& =\left(\begin{array}{l}
\left(y_{1}+y_{2}-x_{1}-x_{2}\right)^{2} \\
\left(y_{1}+y_{2}-x_{1}-x_{2}\right)^{2}
\end{array}\right)
\end{aligned}
$$

we see that $\langle T(y)-T(x), y-x\rangle \in \mathbb{R}_{+}^{2}$, namely, the operator $T$ is $\mathbb{R}_{+}^{2}$-monotone.

For the pseudomonotonicity assumption, let $\langle T(x), y-x\rangle \notin-\mathbb{R}_{+}^{2} \backslash\{(0,0)\}$, namely,

$$
\left(\begin{array}{l}
\left(x_{1}+x_{2}+1\right)\left(y_{1}+y_{2}-x_{1}-x_{2}\right) \\
\left(x_{1}+x_{2}+2\right)\left(y_{1}+y_{2}-x_{1}-x_{2}\right)
\end{array}\right) \notin-\mathbb{R}_{+}^{2} \backslash\{(0,0)\},
$$

which provides that $y_{1}+y_{2}-x_{1}-x_{2} \geq 0$. This, together with

$$
\langle T(y), y-x\rangle=\left(\begin{array}{l}
\left(y_{1}+y_{2}+1\right)\left(y_{1}+y_{2}-x_{1}-x_{2}\right) \\
\left(y_{1}+y_{2}+2\right)\left(y_{1}+y_{2}-x_{1}-x_{2}\right)
\end{array}\right),
$$

assures that $\langle T(y), y-x\rangle \in \mathbb{R}_{+}^{2}$. Therefore, $T$ is pseudomonotone.

Since $T$ is $\mathbb{R}^{2}$-monotone, it implies that the function $\psi$, defined as $\psi(x, y)=\langle T(x), y-x\rangle$, is $\mathbb{R}^{2}$-monotone. Moreover, it is easy to see that bifunction $\psi$ is $\mathbb{R}^{2}$-convex in its second argument. Then, by the proof of [36, Proposition 1], for this $\psi$, we conclude that: for arbitrarily integer $n \geq 1$, for every $x^{1}, \ldots, x^{n} \in K$ and $\lambda_{1}, \ldots, \lambda_{n} \geq 0$ with $\sum_{i=1}^{n} \lambda_{i}=1$, it holds that

$$
\sum_{i=1}^{n}\left\langle T\left(x^{i}\right), \sum_{j=1}^{n} \lambda_{j} x^{j}-x^{i}\right\rangle \in-\mathbb{R}^{2}
$$

whence, hypothesis (iv) of Theorem 4.1 is verified by any functional $c^{*} \in \mathbb{R}^{2^{*}}$. Moreover, $\varphi$ is $\mathbb{R}^{2}$-lower semicontinuous and $\mathbb{R}^{2}$-convex.

It is easy to see that the solution set of the lower level problem is $S_{T}=\{(0,0)\}$. With respect to the constraint set $S_{T}$, the function $\varphi$ admits the pair $(0,0)$ as a Pareto efficient solution, but with respect to the whole set $K$, this point fails to be a Pareto efficient solution because $\varphi(K)-\varphi(0,0)=-K$, and $-K \cap\left(-\mathbb{R}^{2} \backslash\{(0,0)\}\right) \neq \emptyset$. 


\section{RESTRICTED VECTOR SADDLE-POINT PROBLEMS}

One of the most important particular case of $(B S V E P)$ is the strong cone saddle-point problem over an efficient set, which has a key role in the theory of noncooperative games. Vectorvalued payoff functions have been introduced to the game theory in order to describe real-world situations. For the motivation and applications of multicriteria games, we refer to [42, 43]. The Nash equilibrium problem, which was introduced in $[44,45]$ and is related to $n$-person noncooperative games, was extended by Debreu [46] to the generalized Nash equilibrium problem in the way that the strategy of each player may depend on the strategy of the other players. This offers a better description of practical problems arising from economics.

A two player noncooperative vector-valued generalized Nash equilibrium problem is considered. Let $X_{1}$ and $X_{2}$ be nonempty sets, and suppose that the strategy pair allowed for the two players is restricted to a set $S_{\psi} \subseteq X_{1} \times X_{2}$, where $S_{\psi}$ is the set of solutions of the lower level problem:

$$
(S V E P) \text { find } \bar{z} \in X_{1} \times X_{2} \text { such that } \psi(\bar{z}, z) \notin-C \backslash\left\{0_{Y}\right\} \text {, for all } z \in X_{1} \times X_{2} \text {. }
$$

For an arbitrarily strategy pair $\left(x_{1}, x_{2}\right) \in X_{1} \times X_{2}$, consider the strategy sets of the players $x_{2}$ and $x_{1}$ as:

$$
S_{1}\left(x_{2}\right)=\left\{x_{1} \in X_{1} \mid\left(x_{1}, x_{2}\right) \in S_{\psi}\right\}
$$

and

$$
S_{2}\left(x_{1}\right)=\left\{x_{2} \in X_{2} \mid\left(x_{1}, x_{2}\right) \in S_{\psi}\right\} .
$$

Let $\varphi: X_{1} \times X_{2} \rightarrow Y$ be the payoff function of the player 1 and consider the associated zero-sum game. Take into account the sets:

$$
\varphi\left(S_{1}\left(x_{2}\right), x_{2}\right)=\left\{\varphi\left(x_{1}, x_{2}\right) \mid x_{1} \in S_{1}\left(x_{2}\right)\right\}
$$

respectively,

$$
\varphi\left(x_{1}, S_{2}\left(x_{1}\right)\right)=\left\{\varphi\left(x_{1}, x_{2}\right) \mid x_{2} \in S_{2}\left(x_{1}\right)\right\} .
$$

Definition 5.1. A pair $\left(\bar{x}_{1}, \bar{x}_{2}\right) \in S_{\psi}$ is said to be a restricted strong $C$-saddle point for $\varphi$ if

$$
\varphi\left(\bar{x}_{1}, \bar{x}_{2}\right) \in \operatorname{Max}\left(\varphi\left(S_{1}\left(\bar{x}_{2}\right), \bar{x}_{2}\right), C\right) \cap \operatorname{Min}\left(\varphi\left(\bar{x}_{1}, S_{2}\left(\bar{x}_{1}\right)\right), C\right) .
$$

Theorem 5.1. Let $X_{1}$ and $X_{2}$ be convex and compact sets, $c^{*} \in C^{\sharp}$, and suppose that the following conditions are satisfied:

(i) $\varphi$ is properly $C$-upper semicontinuous and $C$-concave in its first argument, properly $C$-lower semicontinuous and $C$-convex in its second argument.

(ii) $\psi(x, x)=0_{Y}$, for all $x \in X_{1} \times X_{2}, \psi$ is properly $C$-lower semicontinuous in its second argument and $C$-antimonotone.

(iii) for all $x, y \in X_{1} \times X_{2}, \psi(x, y) \notin-C \backslash\left\{0_{Y}\right\}$ implies that $\psi(y, x) \in-C$.

(iv) there exists $c^{*} \in C^{\sharp}$ such that $c^{*} \circ \psi$ is essentially quasimonotone.

Then, $\varphi$ has a restricted strong $C$-saddle point.

Proof. Take $K=X_{1} \times X_{2}$ and consider bifunction $\Phi: K \times K \rightarrow Y$ to be defined as:

$$
\Phi(x, y)=\varphi\left(x_{1}, y_{2}\right)-\varphi\left(y_{1}, x_{2}\right), \text { for all } x=\left(x_{1}, x_{2}\right), y=\left(y_{1}, y_{2}\right) \in K \text {. }
$$


Hypothesis (i) assures that $\Phi$ is properly $C$-upper semicontinuous in its first argument and $C$ convex in its second argument. Moreover, $\Phi(x, x)=0$ for all $x \in K$. Since all the assumptions of Theorem 3.1 are satisfied, there exists $\bar{x}=\left(\bar{x}_{1}, \bar{x}_{2}\right) \in X_{1} \times X_{2}$ such that

$$
\Phi(\bar{x}, y) \notin-C \backslash\left\{0_{Y}\right\}, \text { for all } y \in S_{\psi} .
$$

This implies that

$$
\varphi\left(\bar{x}_{1}, y_{2}\right)-\varphi\left(y_{1}, \bar{x}_{2}\right) \notin-C \backslash\left\{0_{Y}\right\} \text {, for all } y=\left(y_{1}, y_{2}\right) \in S_{\psi} .
$$

Setting $y=\left(\bar{x}_{1}, y_{2}\right)$ with $y_{2} \in S_{2}\left(\bar{x}_{1}\right)$, and $y=\left(y_{1}, \bar{x}_{2}\right)$ with $y_{1} \in S_{1}\left(\bar{x}_{2}\right)$ in relation (5.1), we have

$$
\varphi\left(\bar{x}_{1}, y_{2}\right)-\varphi\left(\bar{x}_{1}, \bar{x}_{2}\right) \notin-C \backslash\left\{0_{Y}\right\}, \text { for all } y_{2} \in S_{2}\left(\bar{x}_{1}\right),
$$

and respectively,

$$
\varphi\left(\bar{x}_{1}, \bar{x}_{2}\right)-\varphi\left(y_{1}, \bar{x}_{2}\right) \notin-C \backslash\left\{0_{Y}\right\}, \text { for all } y_{1} \in S_{1}\left(\bar{x}_{2}\right) .
$$

By these relations, we deduce that

$$
\left(\varphi\left(\bar{x}_{1}, S_{2}\left(\bar{x}_{1}\right)\right)-\varphi\left(\bar{x}_{1}, \bar{x}_{2}\right)\right) \cap\left(-C \backslash\left\{0_{Y}\right\}\right)=\emptyset,
$$

and

$$
\left(\varphi\left(S_{1}\left(\bar{x}_{2}\right), \bar{x}_{2}\right)-\varphi\left(\bar{x}_{1}, \bar{x}_{2}\right)\right) \cap\left(C \backslash\left\{0_{Y}\right\}\right)=\emptyset
$$

that is,

$$
\varphi\left(\bar{x}_{1}, \bar{x}_{2}\right) \in \operatorname{Max}\left(\varphi\left(S_{1}\left(\bar{x}_{2}\right), \bar{x}_{2}\right), C\right) \cap \operatorname{Min}\left(\varphi\left(\bar{x}_{1}, S_{2}\left(\bar{x}_{1}\right)\right), C\right) .
$$

Therefore the bifunction $\varphi$ admits a restricted strong $C$-saddle point.

Theorem 5.2. Let $X_{1}$ and $X_{2}$ be convex and compact sets, $c^{*} \in C^{\sharp}$, and suppose that the following conditions are satisfied:

(i) $\varphi$ is $C$-upper semicontinuous and $C$-concave in its first argument, $C$-lower semicontinuous and $C$-convex in its second argument.

(ii) $\psi(x, x)=0$ for all $x \in X_{1} \times X_{2}, \psi$ is $C$-lower semicontinuous in its second argument, $C$-monotone, and, for all $x, y \in X_{1} \times X_{2}$ the function $t \in[0,1] \mapsto \psi(t y+(1-t) x, y)$ is $C$-upper semicontinuous at 0 .

(iii) for all $x, y \in X_{1} \times X_{2}, \psi(x, y) \notin-C \backslash\left\{0_{Y}\right\}$ implies that $\psi(y, x) \in-C$.

(iv) there exists $c^{*} \in C^{\sharp}$ such that $c^{*} \circ \psi$ is essentially quasimonotone.

Then, $\varphi$ has a restricted strong $C$-saddle point.

Proof. Take $K=X_{1} \times X_{2}$ and consider bifunction $\Phi: K \times K \rightarrow Y$ to be defined as:

$$
\Phi(x, y)=\varphi\left(x_{1}, y_{2}\right)-\varphi\left(y_{1}, x_{2}\right), \text { for all } x=\left(x_{1}, x_{2}\right), y=\left(y_{1}, y_{2}\right) \in K .
$$

By hypothesis (i), we deduce that $\Phi$ is $C$-upper semicontinuous in its first argument and $C$ convex in its second argument. Moreover, $\Phi(x, x)=0$ for all $x \in K$. Since all the assumptions of Theorem 3.2 are satisfied, by using a similar reasoning as in the above proof, we have that the bifunction $\varphi$ admits a restricted strong $C$-saddle point.

In the final part of this section, an example with the existence of restricted strong cone saddle points is given. 
Example 5.1. Let $K=[-1,0] \times[-1,0], Y=\mathbb{R}^{2}, C=\mathbb{R}_{+}^{2}, \varphi: K \rightarrow \mathbb{R}^{2}$, and $\psi: K \times K \rightarrow \mathbb{R}^{2}$ be defined as:

$$
\varphi(x)=\left(x_{2}-x_{1}+1, x_{2}-x_{1}\right), \psi(x, y)=\left(y_{1}-x_{1}, y_{1}-x_{1}\right) \text { for all } x=\left(x_{1}, x_{2}\right), y=\left(y_{1}, y_{2}\right) \in K \text {. }
$$

Let $x=\left(x_{1}, x_{2}\right), y=\left(y_{1}, y_{2}\right) \in K$. Evaluating

$$
\psi(x, y)+\psi(y, x)=(0,0) \in-\mathbb{R}_{+}^{2},
$$

we observe that $\psi$ is $\mathbb{R}^{2}$-monotone. Moreover $\psi$ is $\mathbb{R}_{+}^{2}$-lower semicontinuous in its second argument, the function $t \in[0,1] \mapsto \psi(t y+(1-t) x, y)$ is $\mathbb{R}_{+}^{2}$-upper semicontinuous at $0, \psi(x, x)=0$, for all $x \in K$, and $\psi$ is $\mathbb{R}_{+}^{2}$-convex in its second argument. The $\mathbb{R}^{2}$-monotonicity of $\psi$ together with its $\mathbb{R}_{+}^{2}$-convexity in its second argument, according to [36, Proposition 1], they imply that assumption (iv) of Theorem 5.2 is fulfilled.

$$
\begin{gathered}
\psi(x, y)=\left(y_{1}-x_{1}, y_{1}-x_{1}\right) \notin-\mathbb{R}_{+}^{2} \backslash\{(0,0)\} \text { gives } y_{1} \geq x_{1}, \text { whence } \\
\psi(y, x)=\left(x_{1}-y_{1}, x_{1}-y_{1}\right) \in-\mathbb{R}_{+}^{2},
\end{gathered}
$$

and thus, hypothesis (iii) of Theorem 5.2 is verified. Moreover, all the conditions from hypothesis (i) are verified by $\varphi$.

It is easy the see that the solution set of the lower level strong vector equilibrium problem is the set $S_{\psi}=\{-1\} \times[-1,0]$,

$$
S_{1}(-1)=\left\{x_{1} \mid\left(x_{1},-1\right) \in S_{\psi}\right\}=\{-1\}, S_{2}(-1)=\left\{x_{2} \mid\left(-1, x_{2}\right) \in S_{\psi}\right\}=[-1,0],
$$

and respectively,

$$
\varphi\left(S_{1}(-1),-1\right)=\varphi(-1,-1)=(-1,0), \varphi\left(-1, S_{2}(-1)\right)=[-1,0] \times[0,1] .
$$

Hence,

$$
\begin{aligned}
& \left(\varphi\left(S_{1}(-1),-1\right)-\varphi(-1,-1)\right) \cap\left(-\mathbb{R}_{+}^{2} \backslash\{(0,0)\}\right)=\emptyset \\
& \left(\varphi\left(-1, S_{2}(-1)\right)-\varphi(-1,-1)\right) \cap\left(-\mathbb{R}_{+}^{2} \backslash\{(0,0)\}\right)=\emptyset
\end{aligned}
$$

and therefore the pair $(-1,-1)$ is a restricted strong $\mathbb{R}^{2}$-saddle point of $\varphi$.

\section{REFERENCES}

[1] K. Fan, A minimax inequality and applications, in Inequalities III, 103-113. Academic Press, New York, 1972.

[2] L.D. Muu, W. Oettli, Convergence of an adaptive penalty scheme for findind constrained equilibria, Nonlinear Anal. 18 (1992), 1159-1166.

[3] E. Blum, W. Oettli, From optimization and variational inequalities to equilibrium problems, Math. Student 63 (1994), 123-145.

[4] M. Bianchi, S, Schaible, Generalized monotone bifunctions and equilibrium problems, J. Optim. Theory Appl. 90 (1996), 31-43.

[5] S.Y. Cho, A monotone Bregam projection algorithm for fixed point and equilibrium problems in a reflexive Banach space, Filomat, 34 (2020), 1487-1497.

[6] M. Bianchi, R. Pini, A note on equilibrium problems with properly quasimonotone bifunctions, J. Global Optim. 20 (2001), 67-76.

[7] G. Bigi, M. Castellani, G. Kassay, A dual view of equilibrium problems, J. Math. Anal. Appl. 342 (2008), 17-26.

[8] G. Bigi, M. Castellani, M. Pappalardo, M. Passacantando, Existence and solution methods for equilibria, European J. Oper. Res. 227 (2013), 1-11. 
[9] S.Y. Cho, Generalized mixed equilibrium and fixed point problems in a Banach space, J. Nonlinear Sci. Appl. 9 (2016), 1083-1092.

[10] G. Kassay, J. Kolumbán, On a generalized sup-inf problem, J. Optim. Theory Appl. 91 (1996), 651-670.

[11] Q.H. Ansari, W. Oettli, D. Schläger, A generalization of vector equilibria, Math. Meth. Oper. Res. 46 (1997), 147-152.

[12] M. Bianchi, N. Hadjisavvas, S. Schaible, Vector equilibrium problems with generalized monotone bifunctions, J. Optim. Theory Appl. 92 (1997), 527-542.

[13] W. Oettli, A remark on vector-valued equilibria and generalized monotonicity, Acta Math. Vietnam. 22 (1997), 213-221.

[14] Q.H. Ansari, Vector equilibrium problems and vector variational inequalities, In F. Giannessi (ed.) Vector Variational Inequalities and Vector Equilibria, pp. 1-15, Kluver Academic Publishers, Dordrecht 2000.

[15] Q.H. Ansari, J.C. Yao, An existence result for the generalized vector equilibrium problem, Appl. Math. Lett. 12 (1999), 53-56.

[16] Q.H. Ansari, E. Köbis, J.C. Yao, Vector Variational Inequalities and Vector Optimization, Springer, Berlin, 2018.

[17] M. Bianchi, G. Kassay, R. Pini, Ekeland's principle for vector equilibrium problems, Nonlinear Anal. 66 (2007), 1454-1464.

[18] A. Capătă, G. Kassay, On vector equilibrium problems and applications, Taiwanese J. Math. 15 (2011), 365 380.

[19] G. Kassay, V.D. Rădulescu, Equilibrium Problems and Applications, Academic Press, London, 2019.

[20] I.V. Konnov, S. Schaible, Duality for equilibrium problems under generalized monotonicity, J. Optim. Theory Appl. 104 (2000), 395-408.

[21] T. Tanaka, Generalized semicontinuity and existence theorems for cone saddle points, Appl. Math. Optim. 36 (1997), 313-322.

[22] A. Moudafi, Proximal methods for a class of bilevel monotone equilibrium problems, J. Glob. Optim. 47 (2010), 287-292.

[23] B.V. Dinh, L.D. Muu, On penalty and gap function methods for bilevel equilibrium problems, J. Appl. Math 2011 (2011), 1-14.

[24] P.M. Duc, L.D. Muu, A splitting algorithm for a class of bilevel equiibrium problems involving nonexpansive mappings, Optimization 65 (2016), 1855-1866.

[25] V.V. Kalashnikov, N.I. Kalashnikova, Solving two-level variational inequality. Hierarchical and bilevel programming, J. Glob. Optim. 8 (1996), 289-294.

[26] Q. Chadli, Q.H. Ansari, S. Al-Homidan, Existence of solutions and algorithms for bilevel vector equilibrium problems: an auxiliary principle technique, J. Optim. Theory Appl. 172 (2017), 726-758.

[27] A. Göpfert, H. Riahi, C. Tammer, C. Zălinescu, Variational Methods in Partially Ordered Spaces, Springer, New-York, 2003.

[28] D.T. Luc, Theory of Vector Optimization, Springer-Verlag, Berlin, 1989.

[29] W.W. Breckner, G. Orbán, Continuity of generalized convex mappings taking values in an ordered topological linear space, Anal. Numér. Théor. Approx. 11 (1982), 15-33.

[30] G. Bigi, A. Capătă, G. Kassay, Existence results for strong vector equilibrium problems and their applications, Optimization 61 (2012), 567-583.

[31] A.E. Capătă, Contributions to the Theory of Vector and Multifunction Equilibrium Problems, Galaxia Gutenberg, Târgu-Lăpuş, 2010.

[32] B.D. Craven, Mathematical Programming and Control Theory, Chapman and Hall, London, 1978.

[33] M. Castellani, M. Giuli, Ekeland's principle for cyclically antimonotone equilibrium problems, Nonlinear Anal.: Real World Appl. 32 (2016), 213-228.

[34] K. Fan, A generalization of Tychonoff's fixed point theorem, Math. Ann. 142 (1961), 305-310.

[35] A.N. Iusem, G. Kassay, W. Sosa, On certain conditions for the existence of solutions of equilibrium problems, Math. Prog. 116 (2009), 259-273.

[36] G. Kassay, M. Miholca, Existence results for vector equilibrium problems given by a sum of two functions, J. Global. Optim. 63 (2016), 195-211. 
[37] H. Bonnel, J. Morgan, Semivectorial bilevel optimization problem: penalty approach, J. Optim. Theory Appl. 131 (2006), 365-382.

[38] S. Dempe, Bilevel Optimization: Theory, Algorithms, Applications and a Bibliography, In: S. Dempe, A. Zemkoho (eds) Bilevel Optimization. Springer Optimization and Its Applications, vol. 161. Springer, Cham, 2020.

[39] Y.P. Fang, N.J. Huang, Strong vector variational inequalities in Banach spaces, Appl. Math. Lett. 19 (2006), 362-368.

[40] X.H. Gong, Efficiency and Henig efficiency for vector equilibrium problems, J. Optim. Theory Appl. 108 (2001), 139-154.

[41] S. Karamardian, S. Schaible, Seven kinds of monotone maps, J. Optim. Theory Appl. 66 (1990), 37-46.

[42] F. Szidarovszky, M.E. Gershon, L. Duckstein, Techniques for Multi-Objective Decision Making in Systems Management, Elsevier, Amsterdam 1986.

[43] M. Zeleny, Games with multiple payoffs. Internat, J. Game Theory 4 (1975), 179-191.

[44] J.F. Nash, Jr. Equilibrium points in $n$-person games, Proc. Nat. Acad. Sci. USA 36 (1950), $48-49$.

[45] J. Nash, Non-cooperative games, Ann. Math. 54 (1951), 286-295.

[46] G. Debreu, A social equilibrium existence theorem, Proc. Nat. Acad. Sci. USA 38 (1952), 886-893. 